\title{
E.D.E.N.: A tool for the estimation of dose coefficients for non-human biota
}

\author{
K. Beaugelin-Seiller ${ }^{1}$, J. Garnier-Laplace ${ }^{1}$, J .C. Gariel ${ }^{1}$ and F. J asserand ${ }^{2}$ \\ ${ }^{1}$ Institute of Radioprotection and Nuclear Safety, BP. 3, 13115 St. Paul-les-Durance, France \\ ${ }^{2}$ GaliNeo, Millennium Group, 91940 les Ulis, France
}

\begin{abstract}
Current studies within the field of Environmental Risk Assessments associated with the presence of radionuclides in ecosystems tend to implement the usual approach developed for chemicals, that includes four steps: (1) pollutant inventory in the sources of exposure, (2) effect analysis, (3) exposure analysis and (4) risk calculation. In the case of ionising radiation, the third stage involves radiological dose calculation. For humans, this kind of calculation is internationally codified by the ICRP, that provides both models and associated dose coefficients. For non-human biota, this task is a new field of investigations and no international consensus exists at the present time. We thus propose a computer tool to evaluate the dose rate at which the energy produced by ionising radiations is deposited in representative organisms of non-human biota. This energy depends on several parameters, describing the geometry of the relative position of the ionising source and the target, in association with the main properties of the latter. Concerning the source, the radiation type (alpha, beta or gamma) should be taken into account as well as the energy emissions of the concerned nuclides. The target-organism is described by its geometry (assimilated to an ellipsoid characterised by its three axes), its chemical composition and its lifestyle (exposure pathways, dietary habits, behaviour). E.D.E.N. (Elementary Dose Evaluation for Natural environment) is the operational code that we are developing to evaluate the Dose Per Unit of Concentration (expressed in Gy.time $\mathrm{e}^{-1}$ / Bq.mass ${ }^{-1}$ or Bq.volume ${ }^{-1}$ ) due to internal and external exposures of any non-human organism to any radionuclide. A user-friendly interface is provided to build the exposure scenario of interest, characterising the target and the sources involved. Several cases are presented to illustrate the possibilities of E.D.E.N.
\end{abstract}

\section{INTRODUCTION}

Environmental Risk Assessment (ERA) is well codified today for chemicals, even if the methodology, recommended by Europe for example, is still evolving in terms of specifics. The usual approach is based on a four step iterative process: (1) the sources of exposure are characterised in terms of quality and quantity of chemicals involved, (2) the known effects of the identified chemicals are analysed to determine reference values, such as Predicted No Effect Concentrations (PNECs), (3) the exposure situation is investigated to determine Predicted Environmental Concentrations (PECs) and the risk is finally assessed (4) comparing PECs and PNECs. In case of significant risk, the process is re-engaged with refinements at each step.

For radionuclides, a similar approach is currently proposed with the specificity of completing the exposure analysis with adequate radiological dosimetric calculation. As far as humans are concerned, models and data have been - for a long time - internationally approved as recommendations of the International Commission of Radiation Protection. As far as non-human biota are concerned, no such consensus exists, as this kind of calculation is an emergent requirement, associated with the recent 
boom of environmental protection from ionising radiation. If the ERA approaches are quite the same for chemicals and radionuclides, the methodology applied to the latter also needs to be consistent with the human radioprotection system. At present, the basic concept for dosimetric calculation consists in assessing the deposited energy in the target organism, per unit of time.

Taking into account the lack of adapted tools, we propose a method and the corresponding software to evaluate the dose rate by which the energy resulting from ionising radiations is deposited in given living organisms for a number of geometries and exposure scenarios.

Our purpose is illustrated by some case studies, extracted from the FASSET framework, one for the terrestrial environment and one for the freshwater environment, for comparison’s sake.

\section{METHODOLOGY}

\subsection{Objectives}

The way by which the energy emitted by ionising radiations is deposited in any target depends on several criteria. The first one is the source, characterised in terms of quality and quantity. Its localisation with regard to the target organism determines then the exposure nature, internal or external. In both cases, the organism shape and its global chemical composition are also needed. For external exposure, the media composition and the relative position of the organism in its contaminated environment are the last parameters of the problem to be solved. Finally, an operational factor has to be calculated that allows the assessment of the deposited energy for any radionuclide (ie alpha, beta and gamma emitters), both for internal and external exposures, for any organism in any medium. This is the DPUC factor (Dose rate per Unit of Concentration) which is the ratio between the energy deposition rate, expressed in Gy per unit of time, to the radionuclide concentration, expressed in $\mathrm{Bq}$ per unit of mass or volume of the source.

\subsection{Main hypotheses}

As the physical issue of particle transport is a difficult one to deal with, considering the great variation ranges for any of these parameters, some simplifications have to be made. They lead to the two main hypotheses the methodology is built upon. First, any organism is represented as an ellipsoid, defined by its three axes. Second, any source of radiation is homogeneous in terms of chemical composition, density and radionuclide concentration.

Because of their low penetration power, alpha and beta radiations are regarded as negligible in case of external exposure of a considered macroscopic organism.

\subsection{Calculation principles}

When selecting some relevant energies in the ranges corresponding to alpha (1 to $10 \mathrm{MeV}$ ), beta ( 0.3 to $2 \mathrm{MeV}$ ) and gamma (5 keV to $5 \mathrm{MeV}$ ) radiations, the corresponding basic DPUCs are calculated with a dedicated Monte Carlo calculation, that consists in creating and tracking an adequate number of particles. Once uniformly sampled in the source volume with an input energy and a randomly selected direction, each particle is followed: each time it crosses the target organism, the corresponding deposited energy is recorded. For each first selected ray, the whole deposited energy is then distributed in the target volume to determine the basic DPUCs. The energy loss is calculated according to the specificities of each kind of radiation, applying tabulated and calculated stopping power data, respectively for alpha and beta radiations, and mass attenuation coefficients for gamma radiation. The calculation details will be provided in another publication [1]. 
Then, a mathematical interpolation (Lagrange polynomial) based on these basic values determines the DPUC for any other energy value. For a given spectrum, the DPUC corresponding to each ray - called mono-energetic DPUC - can thus be known.

The final operational DPUC for a specified radionuclide is subsequently obtained by the combination of the mono-energetic DPUCs associated with the radionuclide's specific spectrum energies, taking into account their intensity in the spectrum.

\section{E.D.E.N., THE APPLICATION}

Even if some simplifications have been adopted to allow rapid and easier mathematical resolution, the calculation remains complex. In order to make it user-friendly for researchers and assessors, a computer application was developed.

\subsection{Principles}

Based on a common screen lay-out, including a left column menu and an interactive window on the right, the application - available in French as well as in English - was designed to guide the user along the scenario building, before calculating the needed DPUC.

A scenario is a combination of shapes, compositions and sources. To make the application highly flexible, the three elementary components of a scenario are independently defined and are only combined in the last stage before calculation.

All the data other than those specific to the studied case - i.e. mainly nuclear data and associated elements - are tabulated in or calculated by the application.

\subsection{Tool overview}

Besides usual utilities and the summary of the main methodology features, the heart of the application consists of a calculation module, articulated into two parts.

First, the system to study has to be defined, through interactive forms, one for each of the three elementary bricks, according to the criteria previously exposed (cf. §.2.1). The shapes involved, in terms of organisms as well as media, their composition and the radioactive sources (similar screen) to consider can be (1) selected from previously defined lists, (2) updated as much as necessary or (3) created with a brand new description (Figure 1).
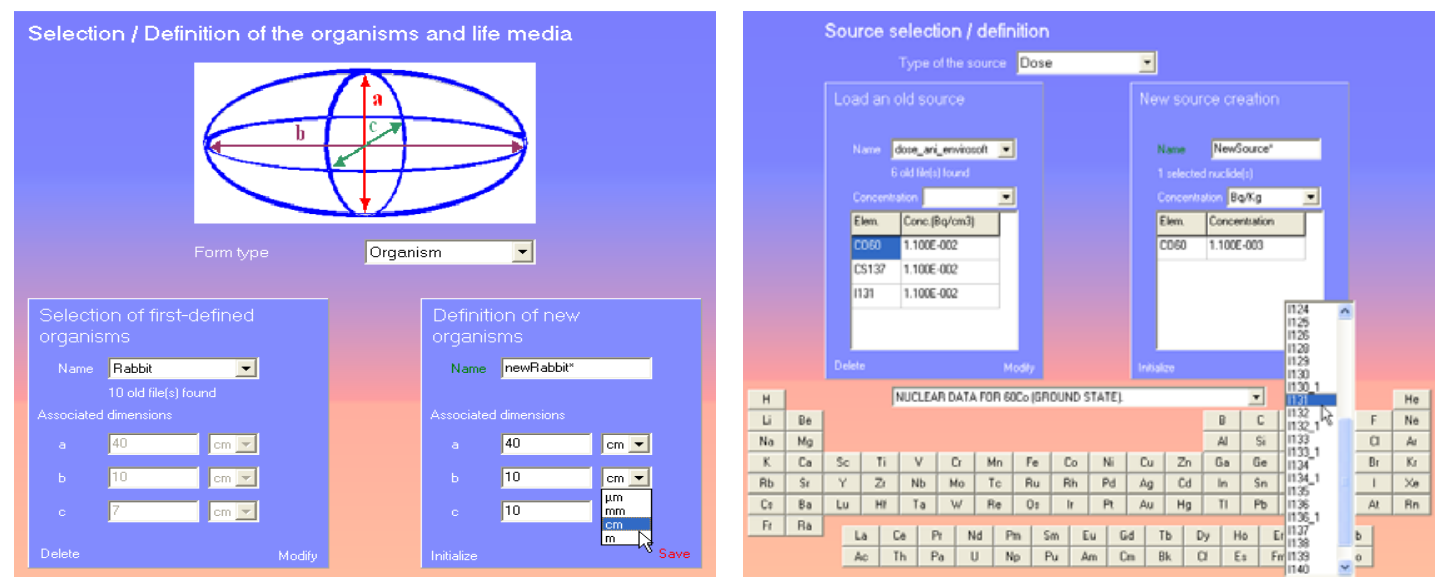

Figure 1. Forms examples (top: shape form, to define an organism; bottom: source form). 


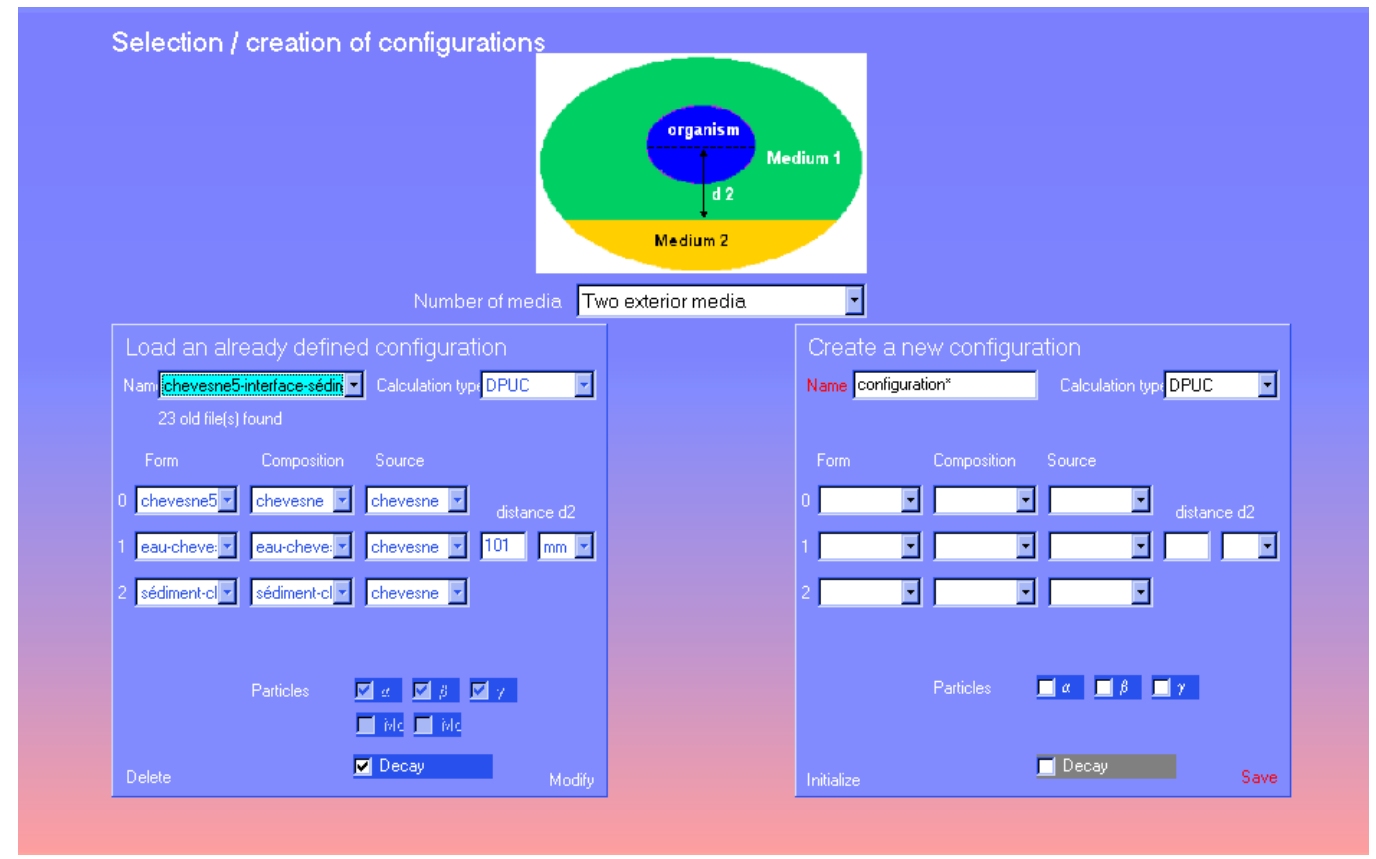

Figure 2. Scenario building screen.

Once chosen, the three blocks are put together within a scenario, which associates to each shape a composition and, if necessary, a source in a given spatial configuration. This screen (Figure 2) also invites the user to specify the desired result (DPUC or dose), the kind of radiation to treat, and allows him to activate - if necessary - the decay option. This last choice allows to take into consideration the daughters of the radionuclides constituting the sources.

Finally, a last screen allows to select all the scenarios to be calculated, and to run them. A control panel then informs the user about the calculation status (time index and process completion).

The output file - compatible with Excel@ - includes a summary of the scenario, the considered decay products - if the corresponding option was activated, messages to help interpret the result, the coding convention for the results and the results themselves. The latter are dispatched in 6 rows, in the following order: nature of result (DPUC or dose), radioisotope of concern, nature of radiation (alpha, beta or gamma), source of concern, numerical value of the result and its unit.

\section{ILLUSTRATIONS}

Two cases studies from FASSET [2] were selected as references to illustrate EDEN calculation. All input data are those of FASSET.

A contaminated freshwater pelagic fish (30x3.5x6 cm, $350 \mathrm{~g})$, externally exposed to contaminated water, was simulated in EDEN. The results are very close for the internal exposure (Figure 3), but differ from about one order of magnitude for the external exposure: depending on the nuclide, EDEN can under- as well as over-estimate the DPUC in relation to the FASSET results. The lack of a constant trend makes it difficult to explain this divergence, that may be due to the FASSET calculation method (empirical analytical formulations versus Monte Carlo calculation for EDEN), and the related solving hypotheses (same density for organism and water, absorbed fraction equal to 1 for alpha radiation, etc.). 
The second simulation concerns a contaminated terrestrial invertebrate $(1 \times 0.03 \times 0.03 \mathrm{~cm}, 740 \mathrm{~g})$ localised in the heart of a $50 \mathrm{~cm}$ contaminated soil layer. FASSET results as well as those of EDEN are obtained with Monte Carlo calculations, but based on different hypotheses, especially concerning the external source description. In spite of these conceptual restrictions, the DPUC calculated are generally very similar for both methodologies, for internal as well as external exposures (Figure 4). The nuclide specificities will be taken into account to thoroughly explain the observed differences.

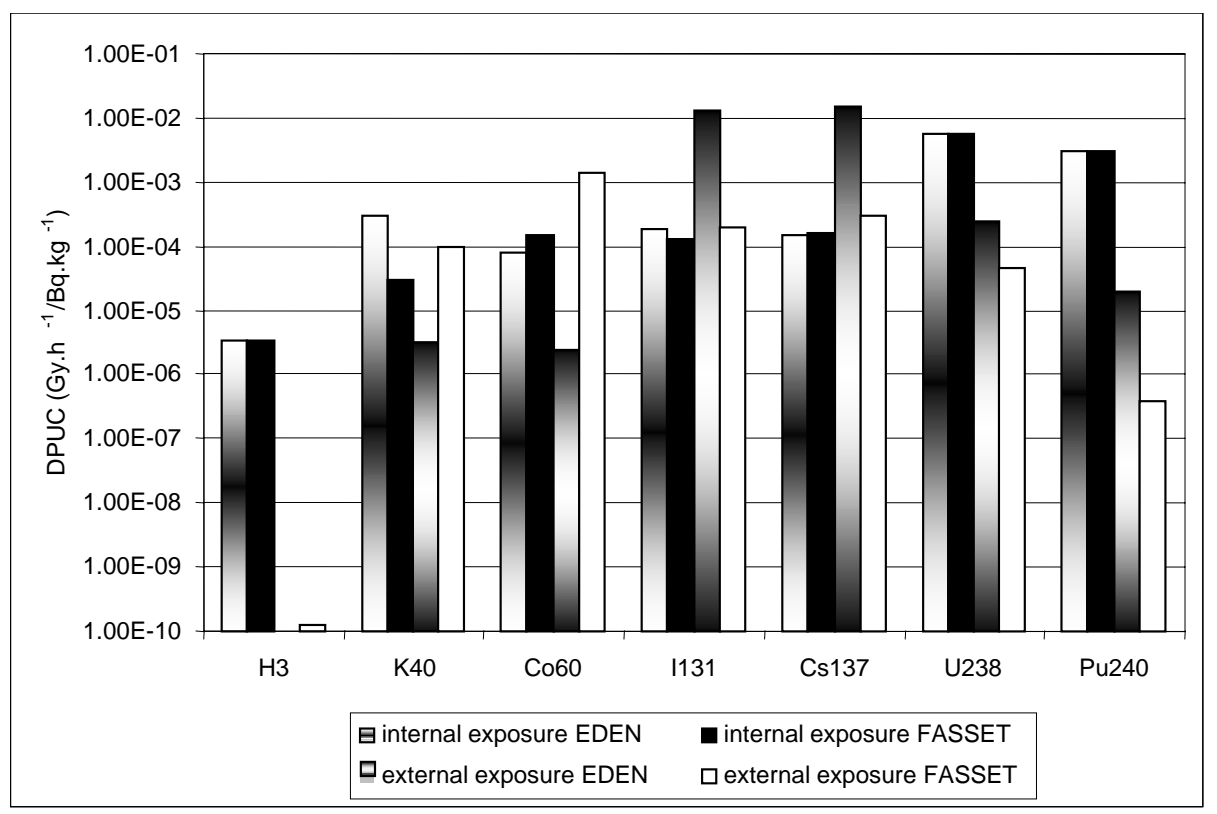

Figure 3. Comparison of results from FASSET and EDEN for the same pelagic freshwater fish.

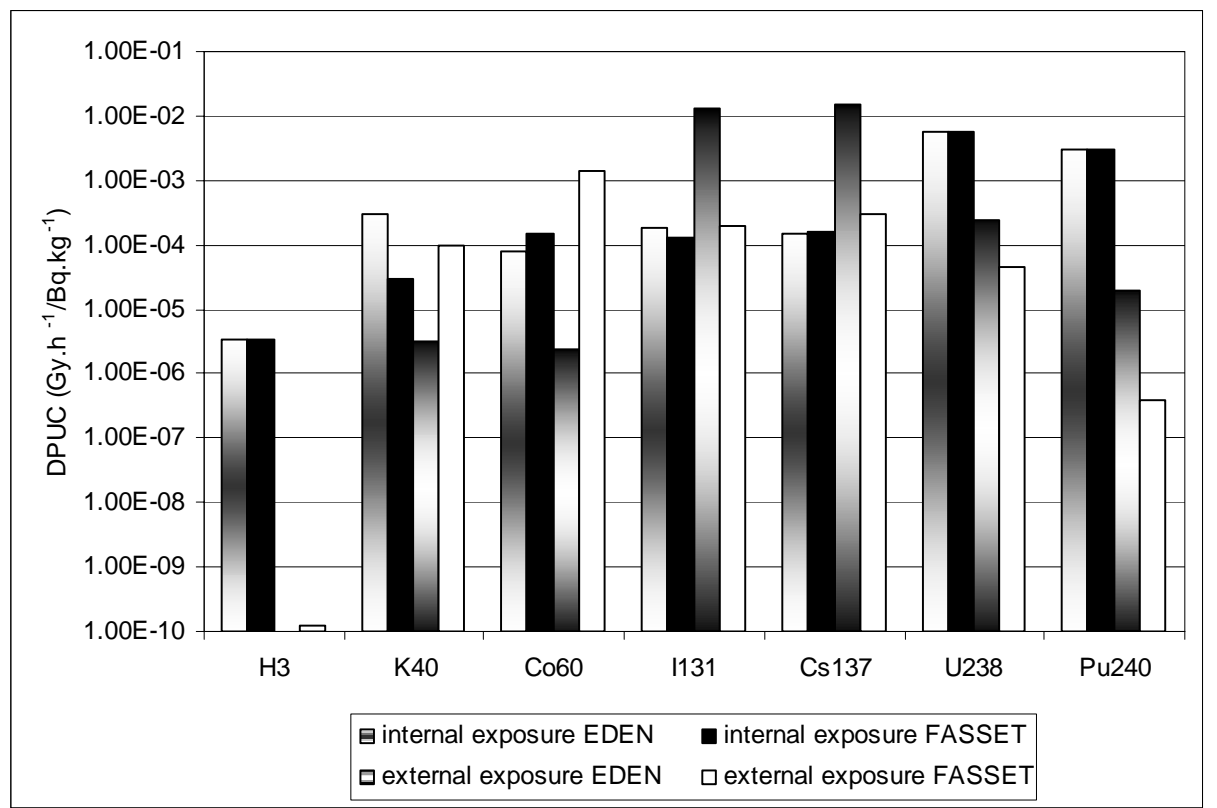

Figure 4. Comparison of results from FASSET and EDEN for the same terrestrial invertebrate. 
Considering their accuracy ( $\pm 10 \%$ ), the results of EDEN are in general adequately close to those obtained in FASSET. When gaps appear, they seem relatively easy to explain in a preliminary stage. But their total understanding and explanation is required, to qualify EDEN. For a comprehensive work, the comparison will have to be extended to other scenarios, so as to cover the great diversity of organisms and situations defined in FASSET.

\section{CONCLUSIONS}

Two ways are offered today to whoever wants to perform a dosimetric calculation for non-human species: heavy physicist calculation codes, long and difficult to operate with, versus empirical analytical approaches, simplistic and accurate in a limited parametric area. Whichever the chosen methodology, its application - until now - has allowed to generate look-up tables or equivalent ([2], [3], [4]), limited to a set of organisms of given sizes, and/or to a set of radionuclides in determined exposure scenarios. If such a rigid approach can be used for the first ERA stage, the risk calculation refinement than can be necessary requires more flexible tools, such as our application. Because it allows dose assessment for any organism exposed to any radionuclide in a user-defined scenario, this kind of flexible, fast and easy-to-use tool appears particularly adapted to environmental radiological risk assessment.

Already in its testing phase, EDEN will have to be compared to several calculation methodologies and, inasmuch as possible, validated on real dose measurements. Its future evolution will have to be linked to the feedback on its use and to its possible interfacing with tools such as CASTEAUR [5] and the whole ERA modelling platform, Symbiose [6].

\section{References}

[1] Beaugelin-Seiller K, Jasserand F, Garnier-Laplace J, Gariel JC. Modelling the radiological dose in non-human species: principles, computerization and application. Health Phys (submitted).

[2] FASSET. Framework for assessment of environmental impact of ionising radiation in major European ecosystems. EC, $5^{\text {th }}$ Framework Program, (available on http://www.fasset.org/ and later on http://www.erica-project.org/ ), 2004.

[3] Environment Agency. Impact Assessment of Ionising Radiation on Wildlife. R\&D Publication 128, Environment Agency, Bristol; 2001.

[4] US-DOE. A graded approach for evaluating radiation doses to aquatic and terrestrial biota. DOE Standard, DOE-STD-1153-2002, US-DOE, Washington; 2002.

[5] Beaugelin-Seiller K, Boyer P, Garnier-Laplace J and Adam C. CASTEAUR: A Simple Tool to Assess the Transfer of Radionuclides in Waterways. Health Phys (2002) 83 (4): 539-542.

[6] Gonze MA, Mourlon C, Garcia-Sanchez L, Beaugelin-Seiller K, Chen T, Le Dizes Maurel S. The Symbiose Project: an Integrated Framework for Performing Environmental Radiological Risk Assessment. ECORAD 2004, Aix-en-Provence, 6-10 septembre 2004. 